1 Hacettepe Journal of Mathematics and Statistics

$\bigcap$ Volume $44(5)$ (2015), $1079-1085$

\title{
On a new reproducing kernel Hilbert space and a boundary value problem for harmonic functions
}

\author{
Alem Memić*
}

\begin{abstract}
In this paper we continue to develop a theory on a new reproducing kernel Hilbert space related to the decomposition theorem for harmonic functions on a domain of the form $\Omega \backslash K$, where $\Omega$ is an open subset of $\mathbb{R}^{n}$ and $K$ a compact subset of $\Omega$.
\end{abstract}

Keywords: harmonic Bergman space, decomposition theorem, harmonically extendable set, integral operator, boundary value problem.

2000 AMS Classification: 31B05, 46E22, 35J25.

Received 15/03/2014 : Accepted 19/06/2014 Doi : 10.15672/HJMS.2015449659

\section{Introduction}

There is a lot of papers about reproducing kernel Hilbert spaces since [1]. This theory found her place also in the area of applied mathematics (see [4]). There are also results about reproducing kernel Hilbert spaces in the framework of real harmonic functions (see [2]) and in the framework of harmonic Bergman spaces (see [3]). In [3] there are explicit formulas for reproducing kernels in the case of a unit ball and a half space. There is no explicit formula for the general case of a reproducing kernels for a harmonic Bergman space on arbitrary domain. In [5] we introduced a new spaces $\mathcal{A}^{p}(\Omega \backslash K)$ of harmonic functions on $\Omega \backslash K$, where $\Omega$ is an open subset of $\mathbb{R}^{n}$ and $K$ is a compact subset of $\Omega$. For these spaces we introduced a new norm and a new inner product (in the case $p=2$ ). Then we obtained a new reproducing kernel for the space $\mathcal{A}^{2}(\Omega \backslash K)$ and found a relation to the standard reproducing kernel on harmonic Bergman space.

This paper is a continuation of [5]. First of all, for an arbitrary nonempty open set $E$ of $\Omega \backslash K$ we introduce a new space $\mathcal{A}^{p}(E)$ and we consider the problem of equalness of $\mathcal{A}^{p}(E)$ and $b^{p}(E)$ and find it's connection to the harmonic extendability. Then we consider the problem of equivalence of norms on the space $\mathcal{A}^{p}(\Omega \backslash K)$. In some cases norms under consideration are equivalent, so we restrict ourselves to those that are equivalent and find some useful properties. For the standard $L^{2}$ inner product on $\mathcal{A}^{2}(\Omega \backslash K)$ we obtain a new reproducing kernel $K_{\Omega \backslash K}$ on $\mathcal{A}^{2}(\Omega \backslash K)$ and prove that this kernel is actually a projection

${ }^{*}$ Department of Mathematics University of Sarajevo, Bosnia and Herzegovina Email: alem.memic@pmf .unsa.ba 
of a kernel $R_{\Omega \backslash K}$ on $\mathcal{A}^{2}(\Omega \backslash K)$. After that, we introduce a new integral operator on $L^{2}(\Omega \backslash K)$ related to the reproducing kernel $S_{\Omega \backslash K}$ and obtain some useful properties. In final, a new kind of a boundary value problem related to the space $\mathcal{A}^{p}(\Omega \backslash K)$ is introduced in the last section. This new boundary value problem is a new type of a boundary value problem for harmonic functions on domains of the form $\Omega \backslash K$. On annular regions we show that this problem has a unique solution. A general case remains open.

\section{Preliminaries}

Let $n \geq 2, \Omega$ an open subset of $\mathbb{R}^{n}$ and $K$ a compact subset of $\Omega$. If $u$ is a harmonic function on $\Omega \backslash K$, there exists functions $v$ and $w$ such that $u=v+w$ on $\Omega \backslash K$, where $v$ is harmonic on $\Omega$ and $w$ is harmonic on $\mathbb{R}^{n} \backslash K$. If we impose condition on $w$ that $\lim _{|x| \rightarrow \infty} w(x)=0$ in the case $n>2$, or $\lim _{|x| \rightarrow \infty} w(x)-\alpha \log |x|=0$ (for some constant $\alpha$ ) in the case $n=2$, then the decomposition $u=v+w$ is unique. The proof of this can be found in [3]. Let $1 \leq p<\infty$. If $E$ is a nonempty open subset of $\mathbb{R}^{n}$, we denote by $b^{p}(E)$ a set of all functions from $L^{p}(E)$ that are harmonic on $E$. This is a Banach space called harmonic Bergman space. More on these spaces can be found in [3]. In [5] we introduced a space $\mathcal{A}^{p}(\Omega \backslash K)$ of all functions $u \in b^{p}(\Omega \backslash K)$ such that $u=v+w$ on $\Omega \backslash K$, where $v \in b^{p}(\Omega)$ and $w \in b^{p}\left(\mathbb{R}^{n} \backslash K\right)$. In [5] we proved that

$$
\mathcal{A}^{p}(\Omega \backslash K)=\left.\left.b^{p}(\Omega)\right|_{\Omega \backslash K} \oplus b^{p}\left(\mathbb{R}^{n} \backslash K\right)\right|_{\Omega \backslash K} .
$$

This is the motivation for the following definition.

2.1. Definition. Let $1 \leq p<\infty, \Omega$ an open subset of $\mathbb{R}^{n}$ and $K$ a compact subset of $\Omega$. Let $E$ be an arbitrary nonempty open subset of $\Omega \backslash K$. We define

$$
\mathcal{A}^{p}(E)=\left.\left.b^{p}(\Omega)\right|_{E} \oplus b^{p}\left(\mathbb{R}^{n} \backslash K\right)\right|_{E} .
$$

2.2. Remark. We should use notation $\mathcal{A}_{\Omega, K}^{p}(E)$ instead of $\mathcal{A}^{p}(E)$ because the previous definition depends also on $\Omega$ and $K$, not just of $E$. We will continue to use notation $\mathcal{A}^{p}(E)$ because $\Omega$ and $K$ will be seen from the context.

2.3. Lemma. For every open set $E$ in $\Omega \backslash K$ it holds

$$
\left.\mathcal{A}^{p}(\Omega \backslash K)\right|_{E}=\mathcal{A}^{p}(E) .
$$

Proof. Let $u \in \mathcal{A}^{p}(E)$. There are $v \in b^{p}(\Omega)$ and $w \in b^{p}\left(\mathbb{R}^{n} \backslash K\right)$ such that $u=v+w$ on $E$. Obviously $v+w$ is harmonic on $\Omega \backslash K$. Let $U=v+w$ on $\Omega \backslash K$. We have $U \in \mathcal{A}^{p}(\Omega \backslash K)$ and $u=\left.U\right|_{E}$, so $\left.u \in \mathcal{A}^{p}(\Omega \backslash K)\right|_{E}$. The other direction is obvious.

In [5] we introduced a problem to find all $(n, p, \Omega, K)$ such that $\mathcal{A}^{p}(\Omega \backslash K)=b^{p}(\Omega \backslash K)$. Here we introduce an analogous problem, to see when $\mathcal{A}^{p}(E)=b^{p}(E)$ for some open set $E$ in $\Omega \backslash K$. We now prove the following theorem.

2.4. Theorem. Let $E$ be a nonempty open subset of $\Omega \backslash K$. Then $\mathcal{A}^{p}(E)=b^{p}(E)$ if and only if $\mathcal{A}^{p}(\Omega \backslash K)=b^{p}(\Omega \backslash K)$ and $\left.b^{p}(\Omega \backslash K)\right|_{E}=b^{p}(E)$.

Proof. Suppose $\mathcal{A}^{p}(E)=b^{p}(E)$. By previous lemma we have $\left.\mathcal{A}^{p}(\Omega \backslash K)\right|_{E}=b^{p}(E)$. Let $u \in b^{p}(\Omega \backslash K)$. Then $\left.u\right|_{E} \in b^{p}(E)=\left.\mathcal{A}^{p}(\Omega \backslash K)\right|_{E}$, so there is $\widetilde{u} \in \mathcal{A}^{p}(\Omega \backslash K)$ such that $\left.u\right|_{E}=\left.\widetilde{u}\right|_{E}$. This and the fact that $u$ and $\widetilde{u}$ are harmonic on $\Omega \backslash K$, implies $u=\widetilde{u}$ on $\Omega \backslash K$. So, $u=\widetilde{u} \in \mathcal{A}^{p}(\Omega \backslash K)$. We conclude that $\mathcal{A}^{p}(\Omega \backslash K)=b^{p}(\Omega \backslash K)$. From this we get $b^{p}(E)=\left.\mathcal{A}^{p}(\Omega \backslash K)\right|_{E}=\left.b^{p}(\Omega \backslash K)\right|_{E}$, so one direction of the theorem is proved. Suppose now that $\mathcal{A}^{p}(\Omega \backslash K)=b^{p}(\Omega \backslash K)$ and $\left.b^{p}(\Omega \backslash K)\right|_{E}=b^{p}(E)$. We have $\mathcal{A}^{p}(E)=$ $\left.\mathcal{A}^{p}(\Omega \backslash K)\right|_{E}=\left.b^{p}(\Omega \backslash K)\right|_{E}=b^{p}(E)$, so the other direction of the theorem also holds, and the proof is finished.

This theorem is a motivation for the following definition. 
2.5. Definition. Let $n \geq 2$ and $1 \leq p<\infty$. Let $\Omega$ be an open subset of $\mathbb{R}^{n}$ and $K$ a compact subset of $\Omega$. We say that a nonempty open subset $E$ of $\Omega \backslash K$ is harmonic $p$-extendable to $\Omega \backslash K$ if $\left.b^{p}(\Omega \backslash K)\right|_{E}=b^{p}(E)$.

So, the last theorem says that $\mathcal{A}^{p}(E)=b^{p}(E)$ if and only if $E$ is a harmonic $p$ extendable to $\Omega \backslash K$ and $\mathcal{A}^{p}(\Omega \backslash K)=b^{p}(\Omega \backslash K)$.

2.6. Corollary. If $\mathcal{A}^{p}(\Omega \backslash K) \neq b^{p}(\Omega \backslash K)$, then $\mathcal{A}^{p}(E) \neq b^{p}(E)$ for every nonempty open subset $E$ of $\Omega \backslash K$.

2.7. Corollary. If $\mathcal{A}^{p}(\Omega \backslash K)=b^{p}(\Omega \backslash K)$, then $\mathcal{A}^{p}(E)=b^{p}(E)$ if and only if $E$ is harmonic p-extendable to $\Omega \backslash K$.

It would be interesting to characterize all harmonic $p$-extendable sets $E$ to $\Omega \backslash K$.

\section{Equivalence of norms}

In [5] we proved the following lemma.

3.1. Lemma. Let $1 \leq p<\infty$ and $u \in \mathcal{A}^{p}(\Omega \backslash K)$ is arbitrarily chosen. Then

$$
\|u\|_{b^{p}(\Omega \backslash K)} \leq 2^{\frac{p-1}{p}}\|u\|_{\mathcal{A}^{p}(\Omega \backslash K)} .
$$

From this lemma we could ask: Is there a $C>0$ such that $\|u\|_{\mathcal{A}^{p}(\Omega \backslash K)} \leq C\|u\|_{b^{p}(\Omega \backslash K)}$ for every $u \in \mathcal{A}^{p}(\Omega \backslash K)$ ?

3.2. Remark. If $\Omega=\mathbb{R}^{n}$ and $K$ an arbitrary compact set of $\mathbb{R}^{n}$, then these norms are equal because $u=v+w$, where $v=0$ on $\mathbb{R}^{n}$. Also, in the case when $K=\{a\}$, where $a \in \Omega$, we have $u=v+w$, where $v \in b^{p}(\Omega), w \in b^{p}\left(\mathbb{R}^{n} \backslash\{a\}\right)=\{0\}$. So, $w=0$ on $\mathbb{R}^{n} \backslash\{a\}$ and $\|u\|_{\mathcal{A}^{p}(\Omega \backslash\{a\})}=\|v\|_{b^{p}(\Omega)}=\|v\|_{b^{p}(\Omega \backslash\{a\})}=\|u\|_{b^{p}(\Omega \backslash\{a\})}$. In both cases we have $C=1$. A general case remains open.

In this section we will consider the case of $(n, p, \Omega, K)$ such that

$$
\|u\|_{\mathcal{A}^{p}(\Omega \backslash K)} \leq C\|u\|_{b^{p}(\Omega \backslash K)}
$$

for every $u \in \mathcal{A}^{p}(\Omega \backslash K)$. This condition with the previous lemma is equivalent that $\|\cdot\|_{\mathcal{A}^{p}(\Omega \backslash K)}$ and $\|\cdot\|_{b^{p}(\Omega \backslash K)}$ are equivalent. So, without further assumption, we suppose that this equivalence of norms is satisfied in the rest of this section.

3.3. Theorem. If $\|\cdot\|_{\mathcal{A}^{p}(\Omega \backslash K)}$ and $\|\cdot\|_{b^{p}(\Omega \backslash K)}$ are equivalent, then $\mathcal{A}^{p}(\Omega \backslash K)$ is a closed subspace of $b^{p}(\Omega \backslash K)$.

Proof. We proved in [5] that $\mathcal{A}^{p}(\Omega \backslash K)$ is a Banach space with respect to $\|\cdot\|_{\mathcal{A}^{p}(\Omega \backslash K)}$. If these norms are equivalent, then $\mathcal{A}^{p}(\Omega \backslash K)$ is a Banach space with respect to $\|\cdot\|_{b^{p}(\Omega \backslash K)}$. Since $b^{p}(\Omega \backslash K)$ is a Banach space with respect to $\|\cdot\|_{b^{p}(\Omega \backslash K)}$ and $\mathcal{A}^{p}(\Omega \backslash K)$ is a Banach space with respect to $\|\cdot\|_{b^{p}(\Omega \backslash K)}$, this implies that $\mathcal{A}^{p}(\Omega \backslash K)$ is a closed subspace of $\|\cdot\|_{b^{p}(\Omega \backslash K)}$ and the proof is finished.

In [5] we proved the following theorem

3.4. Theorem. Suppose $x \in \Omega \backslash K$. Then

$$
|u(x)| \leq \frac{2^{\frac{p-1}{p}}\|u\|_{\mathcal{A}^{p}(\Omega \backslash K)}}{V(B)^{1 / p} d(x, \partial(\Omega \backslash K))^{n / p}}
$$

for every $u \in \mathcal{A}^{p}(\Omega \backslash K)$. 
If we impose condition that the norms $\|\cdot\|_{\mathcal{A}^{p}(\Omega \backslash K)}$ and $\|\cdot\|_{b^{p}(\Omega \backslash K)}$ are equivalent, then we have

$$
|u(x)| \leq \frac{2^{\frac{p-1}{p}} C\|u\|_{b^{p}(\Omega \backslash K)}}{V(B)^{1 / p} d(x, \partial(\Omega \backslash K))^{n / p}}
$$

In the case $p=2$ this means that point evaluation is a bounded linear functional on the Hilbert space $\mathcal{A}^{2}(\Omega \backslash K)$ with respect to $\|\cdot\|_{b^{2}(\Omega \backslash K)}$. This implies that $\mathcal{A}^{2}(\Omega \backslash K)$ is a reproducing kernel Hilbert space. If $x \in \Omega \backslash K$ is arbitrarily chosen, there is a $K_{\Omega \backslash K}(x, \cdot) \in \mathcal{A}^{2}(\Omega \backslash K)$ such that

$$
u(x)=\left\langle u, K_{\Omega \backslash K}(x, \cdot)\right\rangle
$$

for all $u \in \mathcal{A}^{2}(\Omega \backslash K)$ with respect to inner product from $b^{2}(\Omega \backslash K)$.

Because $\mathcal{A}^{2}(\Omega \backslash K)$ is a closed subspace of $b^{2}(\Omega \backslash K)$ and $b^{2}(\Omega \backslash K)$ is a closed subspace of $L^{2}(\Omega \backslash K)$, we have that $\mathcal{A}^{2}(\Omega \backslash K)$ is a closed subspace of the Hilbert space $L^{2}(\Omega \backslash K)$, which implies that there is a unique orthogonal projection of $L^{2}(\Omega \backslash K)$ onto $\mathcal{A}^{2}(\Omega \backslash K)$. We denote this projection by $P_{\Omega \backslash K}$. Let $R_{\Omega \backslash K}$ be a reproducing kernel for $b^{2}(\Omega \backslash K)$. So,

$$
u(x)=\left\langle u, R_{\Omega \backslash K}(x, \cdot)\right\rangle
$$

for every $u \in b^{2}(\Omega \backslash K)$. If we use the fact that $\mathcal{A}^{2}(\Omega \backslash K) \subseteq b^{2}(\Omega \backslash K)$, we get that $K_{\Omega \backslash K}$ is a projection of $R_{\Omega \backslash K}$ to $\mathcal{A}^{2}(\Omega \backslash K)$.

3.5. Theorem. If $x \in \Omega \backslash K$, then

$$
P_{\Omega \backslash K}[u](x)=\int_{\Omega \backslash K} u(y) K_{\Omega \backslash K}(x, y) d y
$$

for all $u \in L^{2}(\Omega \backslash K)$.

Proof. Let $x \in \Omega \backslash K$ and $u \in L^{2}(\Omega \backslash K)$. Then

$$
\begin{aligned}
P_{\Omega \backslash K}[u](x) & =\left\langle P_{\Omega \backslash K}[u], K_{\Omega \backslash K}(x, \cdot)\right\rangle \\
& =\left\langle u, K_{\Omega \backslash K}(x, \cdot)\right\rangle \\
& =\int_{\Omega \backslash K} u(y) K_{\Omega \backslash K}(x, y) d y,
\end{aligned}
$$

where the first equality follows from the reproducing property of $K_{\Omega \backslash K}(x, \cdot)$, the second equality holds because $P_{\Omega \backslash K}$ is a self-adjoint projection onto a subspace containing $K_{\Omega \backslash K}(x, \cdot)$, and the third equality follows from the definition of the inner product and the part 1 . of the following theorem.

3.6. Theorem. The reproducing kernel $K_{\Omega \backslash K}$ has the following properties:

1. $K_{\Omega \backslash K}$ is real valued.

2. If $\left(u_{m}\right)$ is an orthonormal basis of $\mathcal{A}^{2}(\Omega \backslash K)$ with respect to $\|\cdot\|_{b^{2}(\Omega \backslash K)}$, then

$$
K_{\Omega \backslash K}(x, y)=\sum_{m=1}^{\infty} \overline{u_{m}(x)} u_{m}(y)
$$

for all $x, y \in \Omega \backslash K$, where the convergence is pointwise.

3. $K_{\Omega \backslash K}(x, y)=K_{\Omega \backslash K}(y, x)$ for all $x, y \in \Omega \backslash K$.

4.

$$
\left\|K_{\Omega \backslash K}(x, \cdot)\right\|_{b^{2}(\Omega \backslash K)}^{2}=K_{\Omega \backslash K}(x, x)
$$


Proof. 1. Let $u$ be a real valued function from $\mathcal{A}^{2}(\Omega \backslash K)$. Then we have

$$
\begin{aligned}
0=\operatorname{Im}(u(x)) & =\operatorname{Im}\left(\int_{\Omega \backslash K} u(y) \overline{K_{\Omega \backslash K}(x, y)} d y\right) \\
& =-\int_{\Omega \backslash K} u(y) \operatorname{Im}\left(K_{\Omega \backslash K}(x, y)\right) d y
\end{aligned}
$$

If we take $u=\operatorname{Im}\left(K_{\Omega \backslash K}(x, \cdot)\right)$ then we obtain $\int_{\Omega \backslash K}\left(\operatorname{Im}\left(K_{\Omega \backslash K}(x, y)\right)\right)^{2} d y=0$, which implies $\operatorname{Im}\left(K_{\Omega \backslash K}\right) \equiv 0$, so $K_{\Omega \backslash K}$ is real valued.

2. Let $\left(u_{m}(x)\right)$ be any orthonormal basis of $\mathcal{A}^{2}(\Omega \backslash K)$. It exists because of the separability of this space with respect to $\|\cdot\|_{b^{2}(\Omega \backslash K)}$ (norms are equivalent). By standard Hilbert space theory

$$
K_{\Omega \backslash K}(x, \cdot)=\sum_{m=1}^{\infty}\left\langle K_{\Omega \backslash K}(x, \cdot), u_{m}\right\rangle=\sum_{m=1}^{\infty} \overline{u_{m}(x)} u_{m},
$$

where the infinite sum converges in the norm from $b^{2}(\Omega \backslash K)$ restricted to $\mathcal{A}^{2}(\Omega \backslash K)$. Since point evaluation is a continuous linear functional on $\mathcal{A}^{2}(\Omega \backslash K)$, the equation above implies that 2 . holds.

3. This part follows immidiately from 1 . and 2 .

4. Let $x \in \Omega \backslash K$. Then $\left\|K_{\Omega \backslash K}(x, \cdot)\right\|_{b^{2}(\Omega \backslash K)}^{2}=\left\langle K_{\Omega \backslash K}(x, \cdot), K_{\Omega \backslash K}(x, \cdot)\right\rangle=K_{\Omega \backslash K}(x, x)$, where the second equality follows from the reproducing property of $K_{\Omega \backslash K}(x, \cdot)$.

3.7. Remark. In [5], for $x \in \Omega \backslash K$ we introduced a reproducing kernel $S_{\Omega \backslash K}(x, \cdot)$ for a Hilbert space $\mathcal{A}^{2}(\Omega \backslash K)$ with respect to $\|\cdot\|_{\mathcal{A}^{2}(\Omega \backslash K)}$ as a consequence of a boundedness of a linear functional $u \mapsto u(x)$ on $\mathcal{A}^{2}(\Omega \backslash K)$. It is shown in [5] that for $x \in \Omega \backslash K$, $S_{\Omega \backslash K}(x, \cdot)=R_{\Omega}(x, \cdot)+R_{\mathbb{R}^{n} \backslash K}(x, \cdot)$, where $R_{\Omega}(x, \cdot)$ and $R_{\mathbb{R}^{n} \backslash K}(x, \cdot)$ are reproducing kernels for $b^{2}(\Omega)$ and $b^{2}\left(\mathbb{R}^{n} \backslash K\right)$, respectively, obtained as a consequence of boundedness of a linear functional $u \mapsto u(x)$ on these spaces. It would be interesting to see connection between $K_{\Omega \backslash K}$ and $S_{\Omega \backslash K}$.

3.8. Remark. Notations $K_{\Omega \backslash K}$ and $S_{\Omega \backslash K}$ are not good in the sense that in reality these kernels depend on $\Omega$ and $K$, not just on $\Omega \backslash K$. We will use these notations because they are easier to write and we can see what are $\Omega$ and $K$ from the context.

\section{Integral operators}

4.1. Definition. For $u \in L^{2}(\Omega \backslash K)$ we define $M_{\Omega \backslash K}[u]$ by

$$
M_{\Omega \backslash K}[u](x)=\int_{\Omega \backslash K} u(y) S_{\Omega \backslash K}(x, y) d y
$$

for all $x \in \Omega \backslash K$.

4.2. Lemma. If

$$
\int_{\Omega \backslash K} \int_{\Omega \backslash K}\left|S_{\Omega \backslash K}(x, y)\right|^{2} d x d y<\infty,
$$

then $M_{\Omega \backslash K}$ is a bounded linear operator on $L^{2}(\Omega \backslash K)$.

Proof. Linearity is obvious. A boundedness is an immidiate consequence of a Schwartz inequality. 
4.3. Remark. Condition on $S_{\Omega \backslash K}$ in the previous lemma is trivially satisfied in the case $\Omega=\mathbb{R}^{n}$ and $K=\{a\}$ for any $a \in \mathbb{R}^{n}$. It would be interesting to characterize all $\Omega$ and $\mathrm{K}$ such that this condition is satisfied. We can consider also the question on conditions on $\Omega$ and K that imply boundedness of $M_{\Omega \backslash K}$ on $L^{2}(\Omega \backslash K)$.

4.4. Lemma. $M_{\Omega \backslash K}[u]=u$ for all $u \in \mathcal{A}^{2}(\Omega \backslash K)$ if and only if $K_{\Omega \backslash K}(x, \cdot)=S_{\Omega \backslash K}(x, \cdot)$ for every $x \in \Omega \backslash K$.

Proof. " $\Longrightarrow "$. If $M_{\Omega \backslash K}[u]=u$ for all $u \in \mathcal{A}^{2}(\Omega \backslash K)$ then

$$
\int_{\Omega \backslash K} u(y) S_{\Omega \backslash K}(x, y) d y=\int_{\Omega \backslash K} u(y) K_{\Omega \backslash K}(x, y) d y,
$$

for all $x \in \Omega \backslash K$. This implies that $K_{\Omega \backslash K}(x, \cdot)-S_{\Omega \backslash K}(x, \cdot)$ belongs to an orthogonal complement of $\mathcal{A}^{2}(\Omega \backslash K)$ and to the space $\mathcal{A}^{2}(\Omega \backslash K)$ itself. So, it belongs to their intersection and this is a zero set. From this we conclude that $K_{\Omega \backslash K}(x, \cdot)=S_{\Omega \backslash K}(x, \cdot)$.

$" \Longleftarrow "$. This direction follows immidiately from the reproducing property of $K_{\Omega \backslash K}$.

From the fact that $S_{\Omega \backslash K}(x, y)=R_{\Omega}(x, y)+R_{\mathbb{R}^{n} \backslash K}(x, y)$ for all $x, y \in \Omega \backslash K$ (see [5]), we obtain

$$
M_{\Omega \backslash K}[u](x)=\int_{\Omega \backslash K} u(y) R_{\Omega}(x, y) d y+\int_{\Omega \backslash K} u(y) R_{\mathbb{R}^{n} \backslash K}(x, y) d y .
$$

\section{A new type of a boundary value problem}

Let $\Omega$ be an open subset of $\mathbb{R}^{n}$ and $K$ a compact subset of $\Omega$. Suppose $f$ is a continuous function on $\partial \Omega$ and $g$ a continuous function on $\partial K$. Let us consider the following problem. Problem: Can we find a harmonic function $u$ on $\Omega \backslash K$ that is continuous on $\overline{\Omega \backslash K}$ and that has a decomposition $u=v+w$ on $\Omega \backslash K$, where $v$ is a solution to a Dirichlet problem of $\Omega$ with boundary data $f$, and $w$ is a solution to a Dirichlet problem of $\mathbb{R}^{n} \backslash K$ with boundary data $g$ ? Here $v$ and $w$ are from the decomposition theorem for harmonic functions that we consider in this paper. We will call this problem an $(\Omega, K)$ boundary value problem with boudary data $f$ and $g$.

5.1. Definition. For an $(\Omega, K)$ boundary value problem we say it is solvable if for every continuous function $f$ on $\partial \Omega$ and every continuous function $g$ on $\partial K$ there is a solution to the $(\Omega, K)$ boundary value problem with boundary data $f$ and $g$.

5.2. Theorem. Let $n>2,0<r_{0}<r_{1}$. Consider an annular region $\mathbb{A}=\Omega \backslash K$, where $\Omega=\left\{x \in \mathbb{R}^{n},|x|<r_{1}\right\}$ and $K=\left\{x \in \mathbb{R}^{n},|x| \leq r_{0}\right\}$. Then an $(\Omega, K)$ boundary value problem is solvable with a unique solution.

Proof. We will use the following lemma which is a Theorem 4.11 in [3].

5.3. Lemma. Suppose $f \in C(S)$. Then there is a unique function $u$ harmonic on $B^{*}$ and continuous on $\bar{B}^{*}$ such that $\left.u\right|_{S}=f$. Moreover, $u=P_{e}[f]$ on $B^{*} \backslash\{\infty\}$.

If we modify the proof of this lemma we can prove an analogous theorem for arbitrary ball (see exercise 8 in the same chapter). Let us consider now an $(\Omega, K)$ boundary value problem for an annular region $\Omega \backslash K$. Let $f$ and $g$ be a continuous functions on $\partial \Omega=r_{1} S$ and $\partial K=r_{0} S$, respectively, where $S$ is a unit sphere. In this case we obtain a unique solution $v$ to a Dirichlet problem for $\Omega$ with boundary data $f$ and a unique solution $w$ to a Dirichlet problem for $\mathbb{R}^{n} \backslash K$ with boundary data $g$. By the previous lemma $w$ is harmonic at infinity and in the case $n>2$ this is equivalent to the fact that a limit of $w(x)$ is zero when $|x| \longrightarrow \infty$. Let $u=v+w$. Then $u$ is a harmonic function on $\Omega \backslash K$ and a condition at infinity of $w$ is satisfied in the decomposition theorem for harmonic 
functions. Continuity of $v$ on $\bar{\Omega}$ and $w$ on $\overline{\mathbb{R}^{n} \backslash K}$ imply continuity of $u$ on $\overline{\Omega \backslash K}$. We conclude that in the case of an annular region in $\mathbb{R}^{n}$, where $n>2,(\Omega, K)$ boundary value problem is solvable with a unique solution, so the proof is finished.

In general we don't have a solution to $(\Omega, K)$-boundary value problem because the Dirichlet problem is not solvable for an arbitrary open set. If $\Omega$ is a bounded open set and if there is a solution to the Dirichlet problem (here we suppose that the boundary data is a continuous function), then this solution is unique, which is a consequence of a maximum principle for harmonic functions. There are unbounded open sets where we still have a unique solution to a Dirichlet problem, as it is the case for a half space (see chapter 7 in [3]), but in general if a Dirichlet problem is solvable for unbounded regions, we cannot conclude that it is unique because maximum principle for harmonic functions is not satisfied for unbounded regions (see [3]).

5.4. Remark. Let $1 \leq p<\infty$. If $u=v+w$ is a solution to the $(\Omega, K)$ boundary value problem and if $v \in L^{p}(\Omega), w \in L^{p}\left(\mathbb{R}^{n} \backslash K\right)$, then $u \in \mathcal{A}^{p}(\Omega \backslash K)$. It would be interesting to consider the space $\mathcal{A}^{p}(\Omega \backslash K)$ in the framework of this $(\Omega, K)$ boundary value problem for harmonic functions.

5.5. Remark. We could apply these results also in the case of parabolic partial differential equations because there is an analogous decomposition theorem in that case also. (see [6]).

\section{References}

[1] Aronszajn, N. Theory of reproducing kernels, Trans. Amer. Math. Soc. 68, 337-404, 1950.

[2] Auchmuty, G. Reproducing kernels for Hilbert spaces of real harmonic functions, SIAM. J. Math. Anal. 41(5), 1994-2009, 2009.

[3] Axler, S. and Bourdon, P. and W. Ramey, W. Harmonic function theory, Springer-Verlag, New York, 2011

[4] Thomas Hofmann, T. and Schölkopf, B. and Smola, A.J. Kernel methods in machine learning, Ann. Statist. 36(3), 1171-1220, 2008.

[5] Memić, A. A reproducing kernel for a Hilbert space related to harmonic Bergman space on a domain outside compact set, Turk. J. Math. 38, 311-317, 2014.

[6] Watson, N. A., A decomposition theorem for solutions of parabolic equations, Ann. Acad. Sci. Fenn. Math. 25, 151-160, 2001. 
